\title{
Perfiles de ajuste versus vulnerabilidad psicosocial en adolescentes españoles
}

\author{
Psychosocial Adjustment Versus Vulnerability Profiles in \\ Spanish Adolescents
}

\author{
Yolanda Pastor \\ Universidad Rey Juan Carlos, Madrid, España
}

\begin{abstract}
Resumen: El objetivo de este trabajo fue describir los perfiles de ajuste y vulnerabilidad psicosocial en la adolescencia, y estudiar su relación con el malestar emocional en una muestra representativa de jóvenes (52\% varones y $48 \%$ mujeres) de entre 11 y 16 años $(M=13,38 ; D T=1,70)$ de la ciudad de Elche (Alicante, España). Las variables consideradas para la construcción de los perfiles fueron: género, curso académico, dimensiones del autoconcepto, autoestima, apoyo social percibido, cohesión familiar, adaptación escolar, sentimiento de comunidad, cogniciones de hostilidad y problemas de conducta. Se empleó el análisis de conglomerados en dos fases y se realizaron análisis de varianzas y análisis discriminantes para contribuir a la robustez estadística de los resultados, los que revelaron cuatro grupos de adolescentes que variaron en una dimensión, de mayor a menor ajuste psicosocial. Los indicadores de malestar emocional contribuyeron a discriminar entre los grupos, de modo que aquellos con mayor vulnerabilidad psicosocial mostraron mayor malestar emocional. Este trabajo presenta recomendaciones para la detección de adolescentes en riesgo psicosocial, así como para la prevención y diseño de programas.
\end{abstract}

Palabras clave: perfiles psicosociales, adolescencia, malestar emocional, grupos de riesgo.
Abstract: The aim of this paper was to describe profiles of psychosocial adjustment and vulnerability in adolescents, and to study its relationship with emotional distress in a representative sample of young people (52\% boys and $48 \%$ girls) between 11 and 16 years old $(M=13.38 ; S D=$ 1.70) from Elche city (Alicante, Spain). The variables considered for the profiles' construction were: gender, academic level, self-concept dimensions, self-esteem, perceived social support, family cohesion, school adaptation, community feeling, hostile cognitions and behavior problems. A Two-step Clustering Procedure was used and additional variance and discriminant analyses were performed to contribute to the statistical robustness of the results, which revealed four groups of adolescents ranging from higher to lower psychosocial adjustment. Indicators of emotional distress contributed to discriminate between groups, so that groups with higher psychosocial vulnerability showed higher emotional problems. This paper offers recommendations for the detection of adolescents in psychosocial risk, as well as for the prevention and design of programmes.

Keywords: psychosocial profiles, adolescence, emotional distress, risk groups.

Este trabajo ha sido realizado gracias al proyecto de investigación 075/204, financiado por la Escuela Valenciana de Estudios para la Salud, perteneciente a la Conselleria de Sanitat de la Generalitat Valenciana, y dirigido por la autora de este trabajo. La recolección de datos de esta investigación se realizó cuando la autora de este trabajo formaba parte de la plantilla de la Universidad Miguel Hernández de Elche.

Contacto: Y. Pastor. Universidad Rey Juan Carlos, Facultad de Ciencias de la Salud, Edificio departamental II, Despacho 2037. Avda. de Atenas s/n - 28922 Alcorcón, Madrid, España. Correo electrónico: yolanda.pastor@urjc.es

Cómo citar: Pastor, Y. (2016). Perfiles de ajuste versus vulnerabilidad psicosocial en adolescentes españoles. Revista de Psicología, 25(1), 1-21.

http://dx.doi.org/10.5354/0719-0581.2016.41476 


\section{Introducción}

La adolescencia constituye un periodo de transición desde la infancia a la vida adulta, en el que se producen importantes cambios físicos, psicológicos y sociales. Muchos adolescentes atraviesan sin grandes dificultades esta etapa y se convierten en personas adultas saludables. Sin embargo, otros se implican en comportamientos que suponen un riesgo para su salud y desarrollo. De hecho, las principales causas de morbilidad y mortalidad en la adolescencia son, en gran medida, de naturaleza conductual (Feldstein \& Miller, 2006).

Durante décadas los investigadores han tratado de estudiar las interrelaciones entre los diversos comportamientos de riesgo en el periodo adolescente. La literatura ha mostrado que existe una relación positiva entre los distintos comportamientos de riesgo (Feldstein \& Miller, 2006). De modo que algunos autores han postulado la existencia de un síndrome de problemas de conducta en la adolescencia, cuya manifestación depende de la influencia de un conjunto de factores determinantes de naturaleza psicosocial (Jessor, 1993).

En los últimos años, ha surgido un nuevo interés por detectar los grupos de adolescentes de mayor y menor vulnerabilidad al riesgo, y tratar de identificar aquellos factores que influyen esta tendencia. En esta línea, Zweig, Lindberg y McGinley (2001) trataron de resumir perfiles de riesgo, considerando algunas conductas que comprometían la salud (conductas de riesgo sexual, consumo de alcohol, tabaco, marihuana y otras drogas ilícitas, peleas y suicidio).

A partir de una muestra representativa de adolescentes estadounidenses, de entre 14 y 18 años, estos autores detectaron cuatro perfiles de menor a mayor riesgo para ambos géneros con características diferentes. Las variables sociodemográficas (i.e., edad, etnicidad e ingresos familiares) explicaron un pequeño porcentaje de la variabilidad entre los grupos (Zweig et al., 2001). En un trabajo posterior, Zweig, Phillips y Lindberg (2002) encontraron que los grupos de menor riesgo presentaban mejor ajuste psicosocial, adaptación escolar y familiar en ambos géneros.

Usando dos muestras amplias de adolescentes estadounidenses de diferentes décadas (2000 y 1970), Weden y Zabin (2005) detectaron la existencia de grupos de riesgo que se caracterizaban por la presencia de múltiples problemas de conducta; tales como el inicio sexual temprano, el consumo de tabaco, alcohol y marihuana, y el absentismo escolar. Este perfil lo presentaron, en mayor medida, los jóvenes europeoamericanos y de género masculino.

En un trabajo longitudinal de dos cohortes, realizado con adolescentes estadounidenses de entre 12 y 18 años, Bartlett, Holditch-Davis y Belyea (2005) encontraron tres grupos de jóvenes en ambas cohortes que variaron en el tipo y severidad de comportamientos de riesgo. Estos tres grupos fueron denominados: normal, problemático y desviado. Los problemas de aprendizaje y atención, la autoestima y el apoyo paterno y materno, fueron predictores significativos de la pertenencia grupal. El grupo de menos riesgo presentó mayor autoestima en ambas cohortes, mientras que los grupos de mayor riesgo presentaron mayores problemas de atención y aprendizaje, y menos apoyo de ambos padres. Además, se produjeron algunos cambios de pertenencia grupal entre ambas cohortes, encontrando una mayor tendencia hacia el riesgo a medida que aumenta la edad (Bartlett, HolditchDavis, Belyea, Halpern, \& Beeber, 2006). 
El estudio longitudinal realizado por Bryant Ludden y Eccles (2007), con adolescentes estadounidenses de 13 y 16 años, examinó los patrones de consumo de drogas y factores académicos en una muestra de 733 adolescentes de áreas metropolitanas. En primer lugar, los jóvenes de 16 años fueron clasificados en tres grupos, sobre la base del consumo de sustancias (alto, moderado, no consumo); asimismo, fueron clasificados en cuatro grupos (consumidores, iniciadores, desertores y no consumidores), atendiendo a su conducta en las dos edades conjuntamente. Los resultados indicaron que aquellos adolescentes que a los 13 años presentaban mala conducta y bajo rendimiento, tenían mayores probabilidades de pertenecer al grupo de consumidores y de alto consumo en las dos clasificaciones consideradas.

Por su parte, Crockett, Moilanen, Raffaelli y Randall (2006) trataron de relacionar los distintos perfiles psicosociales en los adolescentes entre 12 y 13 años -basándose en rendimiento académico, autorregulación, autovalía y tendencia al riesgo- con la presencia o no de desajustes emocionales cuatro años más tarde. Sus resultados indicaron que los grupos que presentaban ajuste psicosocial mostraban mejor rendimiento escolar, menos problemas de conducta y mayor ajuste emocional cuatro años después. Los grupos de riesgo informaron, en mayor medida, síntomas depresivos, problemas de rendimiento escolar y problemas de conducta cuatro años más tarde.

En España apenas se han realizado estudios que permitan identificar los diferentes perfiles de la población adolescente y que detallen el papel de las características psicosociales y emocionales. En un trabajo descriptivo se presentó una panorámica general de las características psicosociales de los jóvenes españoles de entre 14 y 18 años (Serrano, Godás, Rodríguez, \& Mi- rón, 1996). Los resultados de este trabajo indicaron que los adolescentes españoles se caracterizaban por manifestarse satisfechos con sus relaciones familiares, sus relaciones de amistad y con el entorno escolar. La mayoría de ellos declaró estar adaptados, realizar con frecuencia conductas antisociales leves, pero apenas implicándose en aquellas más graves.

En dicho estudio, la presencia de las conductas antisociales fue mayor en los varones y a medida que aumentaba la edad. El consumo de drogas legales se mostró bastante extendido, pero solo un pequeño porcentaje consumía con regularidad drogas ilegales. El consumo incrementaba con la edad y fue algo mayor para los varones. La baja integración familiar y escolar se relacionó significativamente con la manifestación de conductas antisociales y el consumo de drogas; lo mismo sucedió con el sentimiento de comunidad (Serrano et al., 1996).

En otro trabajo, realizado en Móstoles (Madrid), con una muestra de adolescentes escolarizados de entre 12 y 18 años, Álvarez-Monteserín et al. (1999) describieron tres grupos de adolescentes: uno de preadolescentes de ambos géneros, que presentaron un buen ajuste psicosocial; un segundo grupo, de riesgo, donde predominaron los adolescentes varones mayores de 15 años; $\mathrm{y}$ un tercer grupo, mayoritariamente de adolescentes mujeres, que también mostraron un buen ajuste psicosocial. El grupo de riesgo se caracterizó por un mayor consumo de sustancias, mayor justificación de la violencia, ausencia de motivación hacia el estudio, menor rendimiento académico, baja autoeficacia, peores relaciones familiares y actitudes más racistas e intolerantes que los demás grupos.

Por su parte, Carrasco (2004) trató de identificar grupos en función de sus hábi- 
tos de salud, la satisfacción escolar y el apoyo familiar, a partir de una muestra representativa de adolescentes españoles escolarizados entre los 11 y 17 años. Los grupos de menor edad (adolescentes de ambos géneros de 11 años y varones de 13 años) mostraron hábitos saludables, confianza en sí mismos, satisfacción escolar y buena percepción de apoyo familiar. Sin embargo, las adolescentes de 13 años se caracterizaron por ser sedentarias y presentar baja confianza en sí mismas, mayor presión escolar, decaimiento, síntomas psicosomáticos, dificultad para hacer amigos/as íntimos/as y problemas en la comunicación con sus padres.

A medida que aumentaba la edad (15 y 17 años) se identificaron grupos con estilos de vida más insanos (consumo de tabaco y alcohol), en los que el género jugó un papel fundamental para discriminar entre ellos. Así, las adolescentes presentaron, además, una vida sedentaria, trastornos en los hábitos alimentarios, frecuentes trastornos psicosomáticos, decaimiento, insatisfacción con su físico y escasa confianza en sí mismas. En contraposición, los varones mostraron una confianza elevada y mayor sociabilidad, tenían muchos/as amigos/as y no les gustaba la escuela (Carrasco, 2004).

En otro estudio, realizado con jóvenes de San Sebastián de entre 12 y 20 años, se trató de dibujar una tipología de los adolescentes en función de tres variables: actividades fuera del horario escolar, valores y relaciones familiares. Los resultados definieron seis grupos: normativos $(20,1 \%)$, conflictivos sociales $(7,9 \%)$, familiares liberales $(26,3 \%)$, conflictivos familiares $(7,8 \%)$, ventajistas $(17,9 \%)$ y afamiliares $(20,1 \%)$. Las adolescentes y los de menor edad fueron incluidos con mayor frecuencia en los grupos normativos y menos conflictivos. Estos grupos parecían conformarse en torno a dos ejes, uno de mayor a menor integración social, y otro, de mejor a peor calidad de las relaciones familiares (Elzo Imaz, 2005).

En resumen, la evidencia empírica ha puesto de manifiesto que el género y la edad son variables centrales en distinguir entre grupos de adolescentes. Así, los de mayor edad y de género masculino pertenecen con mayor frecuencia a grupos de mayor riesgo psicosocial y conductual; mientras que las adolescentes muestran, en mayor medida, malestar emocional. Estos hallazgos son consistentes con los resultados ampliamente informados en la literatura acerca de la mayor presencia de problemas externalizantes en los varones e internalizantes en las mujeres (Kramer, Krueger, \& Hicks, 2008).

Sin embargo, son pocos los trabajos realizados con muestra española que incorporen diversas variables psicosociales para encontrar perfiles de mayor riesgo y vulnerabilidad, que permitan discriminar a los jóvenes que se implican en conductas de riesgo y los que no. Estos perfiles permitirían, además, identificar aquellas áreas en las que hay que intervenir para prevenir o reducir la cantidad de jóvenes que se implica en conductas de riesgo. Algunas de estas variables psicosociales son bien conocidas y estudiadas.

La autoestima constituye uno de los mejores indicadores de salud mental y bienestar subjetivo (para una revisión, véase Sedikides \& Gregg, 2003), y ha mostrado, repetidamente en la literatura científica, una moderada relación negativa con conductas de riesgo y problemas de conducta en los jóvenes (Donnellan, Trzesniewski, Robins, Moffitt, \& Caspi, 2005; Jackman $\&$ MacPhee, 2015).

Algunas dimensiones del autoconcepto también han aparecido asociadas al con- 
sumo de sustancias, así, la competencia conductual y la escolar se relacionan negativamente con el consumo de alcohol, tabaco y cannabis en adolescentes; mientras que las competencias relacionadas con las relaciones sociales se asocian positivamente (Pastor, Balaguer, \& GarcíaMerita, 2006, 2008).

La familia es otro de los pilares importantes para el buen desarrollo de los adolescentes (Gavazzi, 2013). El grado de cohesión familiar puede jugar un rol importante en el desarrollo de conductas de riesgo en la adolescencia. Altos niveles de cohesión familiar constituyen un factor protector del consumo de sustancias (Vakalahi, 2001) e, incluso, han aparecido asociados a menores problemas futuros con el consumo de alcohol en la adolescencia (Reeb et al., 2015).

La carencia de apoyo social en la adolescencia ha sido considerada, en uno de los últimos informes publicados por la Organización Mundial de la Salud, como uno de los factores de riesgo más importantes para el desarrollo de trastornos mentales (Mathieson \& Koller, 2008). Algunos estudios indican que el apoyo social constituye un factor protector frente al consumo de sustancias (Jiménez, Musitu, \& Murgui, 2006) o conductas delictivas (Jiménez Gutiérrez, Musitu Ochoa, \& Murgui Pérez, 2005), constituyendo un recurso fundamental para la adhesión y mantenimiento de conductas saludables (Kelder, Hoelscher, \& Perry, 2015).

Asimismo, la adaptación al entorno escolar es clave en el bienestar y desarrollo óptimo de los adolescentes (para una revisión véase Little, Akin-Little, \& Lloyd, 2013). Estudios actuales muestran que los adolescentes que se sienten bien en la escuela y tienen actitudes positivas hacia el profesor, tienen menor riesgo de con- sumo de sustancias (Trenz, Dunne, Zur, \& Latimer, 2015) y de problemas conductuales (Welsh \& Harding, 2015).

Por último, está bien establecido que las características de la comunidad constituyen un potente factor protector o de riesgo para el desarrollo adolescente (ReitzKrueger, Nagel, Guarnera, \& Reppucci, 2015). De la pertenecía a la misma, se desprende el sentimiento de comunidad, el cual constituye un indicador del grado de integración social de la persona en su entorno social. El sentimiento de comunidad positivo se asocia a un menor consumo de sustancias (Mayberry, Espelage, \& Koenig, 2009).

Aunque los resultados de estos trabajos resultan clarificadores, son necesarios estudios adicionales que busquen identificar los distintos perfiles de ajuste, versus vulnerabilidad al riesgo, en población adolescente española; que analicen tanto el papel de las características psicosociales, como sus repercusiones en la salud emocional de los jóvenes. Así, el primer objetivo de este trabajo fue identificar los grupos naturales que surgen en la adolescencia, atendiendo a sus características sociodemográficas (edad y género), psicosociales y conductuales. El segundo objetivo consistió en examinar si los indicadores de malestar emocional contribuyen a discriminar entre los grupos encontrados.

En consonancia con estudios previos, se hipotetiza que: 1) el curso académico (edad) y el género contribuyen a discriminar entre grupos de mayor a menor ajuste psicosocial; 2) a medida que aumenta el curso (la edad) disminuye el ajuste psicosocial y aumenta la probabilidad de pertenecer a grupos de riesgo; 3 ) las adolescentes presentan una autoestima y autoconcepto más bajo y mayor males- 
tar emocional, mientras que los adolescentes presentan mayores problemas de conducta; y 4) los grupos de menor ajuste psicosocial manifiestan mayor malestar emocional.

\section{Método}

\section{Participantes}

Para la selección de la muestra, se utilizaron los datos del censo de los centros docentes de la Generalitat Valenciana (2012), recurriendo a tres criterios de estratificación: el curso académico $\left(6^{\circ}\right.$ primaria, $2^{\circ}$ y $4^{\circ}$ de educación secundaria obligatoria), la distribución administrativa del término municipal de Elche (Alicante, España) (área metropolitana y no metropolitana) y el tipo de centro (público y concertado). Los tamaños muestrales han sido obtenidos utilizando un muestreo aleatorio simple para cada una de las variables de estratificación, teniendo en cuenta las desviaciones típicas de las variables en un estudio piloto. Se asumió un error máximo de estimación de 0,09 puntos y un nivel de confianza de 0,95 .

El total de adolescentes encuestados fue de 648, siendo eliminados aquellos casos donde el porcentaje de datos perdidos fue superior al $5 \%$. Con la muestra restante, se sustituyeron los datos ausentes con la media en el ítem. Por tanto, la muestra final se compuso de 615 adolescentes (52\% varones y $48 \%$ mujeres) de 11 a 16 años $(M=13,38 ; D T=1,70)$ del término municipal de Elche, que cursaban sus estudios en $6^{\circ}$ de primaria $(M$ edad $=$ $11,22 ; D T=0,56), 2^{\circ}$ de educación secundaria obligatoria $(M$ edad $=13,35 ; D T$ $=0,63)$ y $4^{\circ}$ de educación secundaria obligatoria $(M$ edad $=15,21 ; D T=0,52)$.

\section{Variables e instrumentos}

Las variables consideradas fueron: sociodemográficas (género y curso académico), conductuales (problemas de conducta y cogniciones de hostilidad) y psicosociales (autoconcepto, autoestima, apoyo social percibido, cohesión familiar, adaptación escolar y sentimiento de comunidad). Las variables utilizadas para evaluar el malestar emocional fueron la autoculpa, ideación suicida, ánimo depresivo y falta de energía/interés.

La batería de cuestionarios incluyó ítems elaborados ad hoc para evaluar las variables sociodemográficas. Los problemas de conducta fueron evaluados con la Escala de Problemas de Conducta Externalizantes, adaptación española (Estévez et al., 2004) del Externalizating Problem Behavior (Stattin, Romelsjö, \& Stenbacka, 1997). El Externalizating Problem Behavior es un cuestionario de 14 ítems que valora si el adolescente ha cometido alguna vez un acto delictivo, vandálico o ha consumido drogas. La escala de respuesta oscila entre $1=$ nunca y $5=$ más de 10 veces.

Los análisis factoriales exploratorios de este instrumento, han mostrado la presencia de dos factores: Problemas de conducta graves, como participar en actos vandálicos graves, robos con allanamiento y coacción, robos de vehículos, no pagar lo que consumes o herir gravemente a alguien; y Problemas de conducta leves, que hace referencia a conductas tales como el consumo de hachís, emborracharse, hacer grafiti, pequeños hurtos en supermercados o participar en peleas. Ambas escalas presentaron una adecuada consistencia interna en el presente trabajo de ,90 y ,86 respectivamente. 
Las cogniciones de hostilidad fueron evaluadas a partir de un instrumento, de elaboración propia, de seis ítems que recogen pensamientos de rabia y hostilidad hacia los demás, con una escala de respuesta Likert de 5 puntos que oscila entre casi nunca (1) a casi siempre (5). Los ítems de esta escala saturan en un único factor (saturaciones $>, 60)$ y poseen consistencia interna (alfa de Cronbach $=, 83$ ).

El autoconcepto y la autoestima fueron evaluados con una adaptación del Perfil de Autopercepciones (Harter, 1985a), que consta de cinco dimensiones del autoconcepto: competencia académica, competencia deportiva, apariencia física, aceptación social y adecuación de la conducta; y una subescala sobre autoestima o autovalía global. La validez factorial de este instrumento ha sido confirmada con población española (Atienza, Balaguer, \& Moreno, 2002). La consistencia interna de las subescalas en nuestra muestra varió de ,70 a ,83.

El apoyo social percibido se evaluó con la Escala de Apoyo Social (Harter, 1985b), que consta de cuatro subescalas: padres, compañeros de clase, amigos y profesores. La validez factorial de esta escala ha sido confirmada con población española (Pastor, Quiles, \& Pamies, 2012). La consistencia interna de las subescalas en la muestra del presente estudio osciló entre ,70 y, 88 .

La cohesión familiar fue evaluada a través de la subescala de Olson, Portner y Lavee (1987) sobre evaluación del sistema familiar. Esta subescala consta de 10 ítems con una escala de respuesta Likert de 5 puntos, que varía entre casi nunca (1) a casi siempre (5). Este instrumento presenta una adecuada validez y fiabilidad con población española (Musitu, Buelga, Lila, \& Cava, 2001). El coeficiente de consis- tencia interna en el presente estudio fue de, 86 .

La adaptación escolar fue evaluada con seis ítems de elaboración propia que responden a la pregunta "¿Cómo te encuentras en la escuela?", con la misma escala de respuesta que el anterior cuestionario. Los ítems de esta escala saturan en un único factor (saturaciones > ,65) y su consistencia interna es ,81.

La Escala de Sentimiento de Comunidad ha sido extraída del cuestionario The Neighborhood Cohesion Instrument $(\mathrm{Bu}-$ ckner, 1988, adaptación Serrano et al., 1996). Consta de 18 ítems, encabezados por la pregunta "¿Estás satisfecho con tu lugar de residencia?", con una escala de respuesta Likert de 5 puntos, que varía entre muy en desacuerdo (1) a muy de acuerdo (5). Los análisis factoriales exploratorios muestran que los ítems saturan en un único factor (saturaciones > ,30), siendo la consistencia interna (alfa de Cronbach) superior a ,90.

Por último, el malestar emocional fue medido mediante una adaptación española del Perfil de Dimensiones de Depresión (Harter \& Nowakowski, 1987). La validez factorial de esta escala ha sido confirmada con población española (Benavides et al., 2004) y la consistencia interna de las subescalas en el presente estudio osciló entre $, 73 \mathrm{y}, 84$.

\section{Procedimiento}

Se solicitó permiso por carta a cada uno de los centros docentes seleccionados aleatoriamente para participar en la investigación. La administración de cuestionarios tuvo lugar durante el horario escolar. Dos investigadores estuvieron presentes en cada aplicación grupal para explicar las características de los instrumentos y 
resolver las dudas surgidas. Los participantes obtuvieron el consentimiento por escrito de sus padres para participar en la investigación. Se garantizó el anonimato, tanto de los centros como de los jóvenes que participaron en el estudio.

\section{Análisis estadísticos}

Para identificar las agrupaciones naturales que se producen en la muestra de adolescentes, se utilizó el análisis de conglomerados en dos fases, esto por dos razones: primero, por el tamaño de la muestra, dado que cuando se trata de muestras superiores a 200 se recomienda métodos no jerárquicos; y segundo, por la inclusión de variables categóricas y continuas.

Dado que los resultados de esta prueba dependen del orden de los casos, para garantizar la fiabilidad se llevaron a cabo 10 repeticiones de estos análisis, alterando aleatoriamente el orden de los casos. Los resultados obtenidos fueron replicados en todas las repeticiones realizadas. Para garantizar la validez de los resultados, se llevó a cabo un análisis multivariante de la varianza (MANOVA, por su nombre en inglés Multivariate analysis of variance) en el que se utilizó la asignación a los conglomerados como variable independiente, y las variables empleadas en la formación de los mismos, como variables dependientes. Dado que la investigación se inició a partir de cuatro perfiles o conglomerados, se llevaron a cabo pruebas de Bonferroni con el objetivo de detectar entre cuáles de estos existían diferencias significativas en las distintas variables consideradas.

A continuación, se calculó una puntuación total de ajuste psicosocial, la cual consistió en la media de todas las variables conductuales y psicosociales consideradas. Se realizó un análisis de varianza
(ANOVA, por su nombre en inglés Analysis of variance), utilizando como variable dependiente el ajuste psicosocial, y como variable independiente la asignación de los sujetos a los cuatro grupos, con el fin de analizar las diferencias entre ellos en ajuste psicosocial, y valorar si era posible ordenarlos en función del grado de dicho ajuste.

Por último, para estudiar si los indicadores de malestar emocional discriminaban entre los grupos encontrados, se utilizaron dos pruebas estadísticas: el MANOVA, con objeto de valorar si las cuatro variables consideradas eran relevantes; y el análisis discriminante descriptivo, con aquellas variables que fueron significativas en el análisis previo. El análisis de datos fue realizado con el paquete estadístico SPSS 16.0.

\section{Resultados}

Detección de perfiles de ajuste versus vulnerabilidad psicosocial en la adolescencia

La solución de cuatro conglomerados reveló un valor más bajo del Criterio de Información Bayesiano (BIC, por su nombre en inglés Bayesian Information Criterion) $(\mathrm{BIC}=7802,24)$ que la solución de tres conglomerados $(\mathrm{BIC}=8007,49)$, así como un valor alto de la Razón de Cambios (RC) en $\mathrm{BIC}\left(\mathrm{RC}^{\mathrm{BIC}}=, 29\right)$ y en la Razón de Medias de Distancia $(\mathrm{RMD})(\mathrm{RMD}=1,50)$. Estos resultados indicaron que la solución de cuatro conglomerados constituía una agrupación plausible de la muestra.

La distribución de los adolescentes en cuatro conglomerados $(\mathrm{C} 1, \mathrm{C} 2, \mathrm{C} 3$ y $\mathrm{C} 4)$ fue de 171, 159, 203 y 82, respectivamente. Atendiendo a las características de curso y género. La tabla 1 presenta la composición de los conglomerados identificados. 
Tabla 1

Composición de los conglomerados en función de las características de curso y género

\begin{tabular}{lccccc}
\hline & $\mathrm{C} 1$ & $\mathrm{C} 2$ & $\mathrm{C} 3$ & $\mathrm{C} 4$ & \multirow{2}{*}{$\eta^{2}$} \\
\cline { 2 - 5 } & \multicolumn{5}{c}{ Frecuencia (\%) } \\
\hline Género \\
Varones & $85(26,72)$ & $159(50,00)$ & $0(0,00)$ & $74(23,38)$ & \\
Mujeres & $86(29,00)$ & $0(0,00)$ & $203(68,41)$ & $8(2,69)$ & \\
Curso académico & & & &, 67 \\
$6^{\circ}$ Primaria & $171(94,47)$ & $0(0,00)$ & $0(0,00)$ & $10(5,53)$ & \\
$2^{\circ}$ Secundaria & $0(0,00)$ & $78(35,61)$ & $116(53,00)$ & $25(11,39)$ \\
$4^{\circ}$ Secundaria & $0(0,00)$ & $81(37,68)$ & $87(40,46)$ & $47(21,86)$ \\
\hline
\end{tabular}

Nota: $\eta^{2}=$ Eta cuadrado.

Los resultados del MANOVA indicaron un efecto multivariado significativo (Traza de Pillai $=1,93 ; F(54,930)=60,09 ; p$ $\left.<, 001 ; \eta^{2}=, 64\right)$. Los análisis univariados mostraron efectos significativos para todas las variables sociodemográficas, conductuales y psicosociales consideradas, en todas $p<, 001$, excepto para la variable aceptación social $(p<, 05)$.

El género y el curso fueron las variables que mayor peso mostraron para diferenciar entre los cuatro grupos $\left(\eta^{2}=, 67\right.$ para cada una) de entre todas las variables dependientes consideradas (sociodemográficas, conductuales y psicosociales). Las comparaciones con corrección de Bonferroni mostraron diferencias significativas entre los diferentes grupos en todas las variables estudiadas.

El peso de las distintas variables conductuales y psicosociales en la formación de los conglomerados queda reflejado en los gráficos 1 a 4, donde el orden que presentan las variables indica el grado de importancia de las mismas en la formación del conglomerado y, asimismo, se indica gráficamente si la variable es significativa $(t$ de Student).
La figura 1 muestra que los y las adolescentes de $6^{\circ}$ de primaria (C1), de 11 años, se caracterizan por estar significativamente por debajo de la media en la frecuencia de problemas conductuales graves y leves; así como por encima de la misma en adaptación escolar, cohesión familiar, apoyo social percibido de los padres, sentimiento de comunidad, autoestima, competencia académica, apariencia física y apoyo social percibido de los profesores. Además, este grupo se situó por debajo de la media, aunque con menor importancia, en cogniciones de hostilidad. Por su composición y características, a este grupo se le denominó "adolescentes de $6^{\circ}$ de primaria adaptados".

Siguiendo la figura 2, los adolescentes varones de $2^{\circ}$ y $4^{\circ}$ de secundaria (C2), de 13 y 15 años respectivamente, se caracterizan por estar significativamente por debajo de la media en la presencia de problemas de conducta graves y leves, y por encima de la misma en sus percepciones acerca de la adecuación de la conducta, la competencia deportiva, el apoyo social de los padres y la apariencia física. Este grupo fue etiquetado como "varones de secundaria adaptados". 


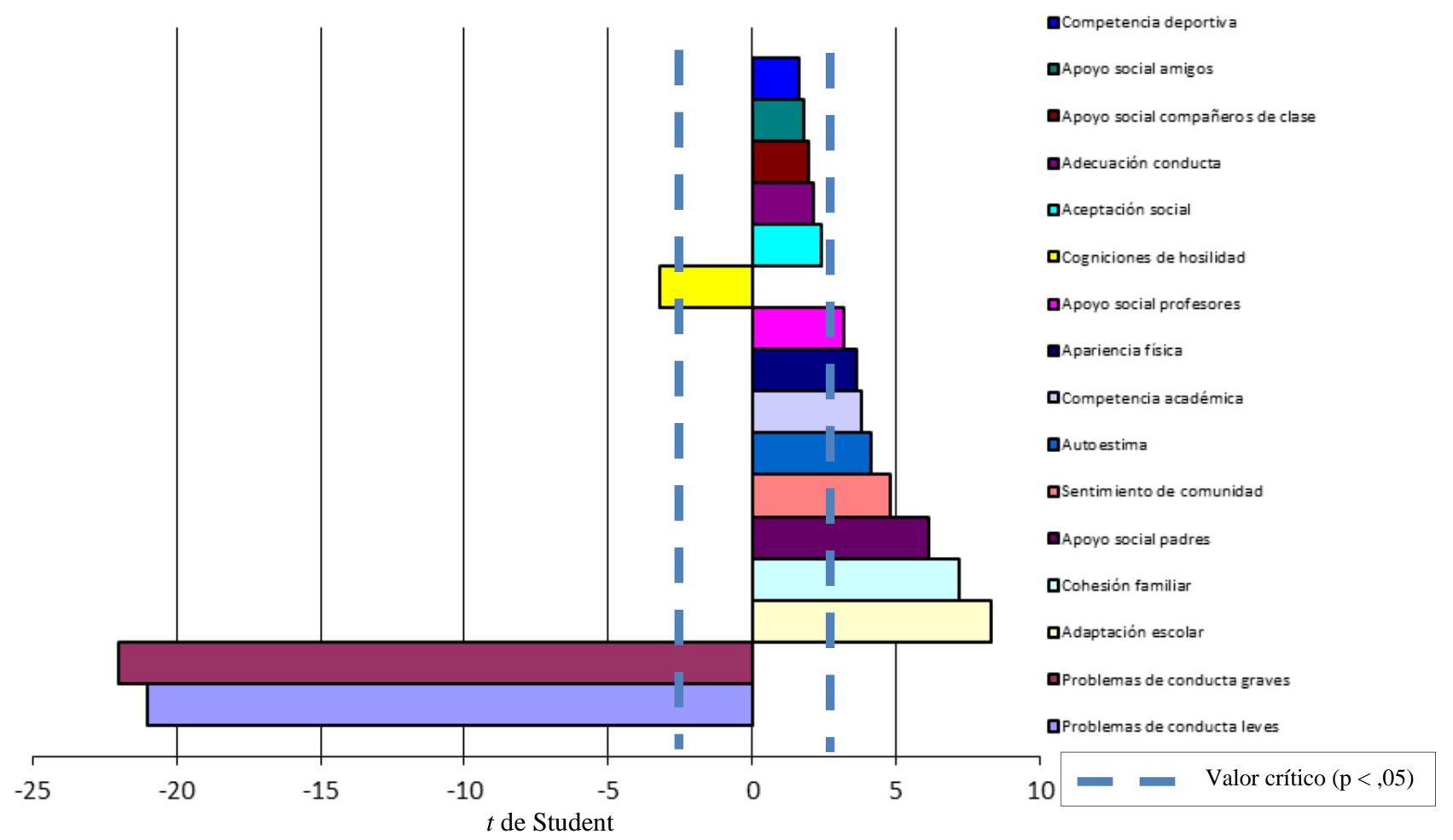

Figura 1. Adolescentes de $6^{\circ}$ de primaria adaptados (C1).

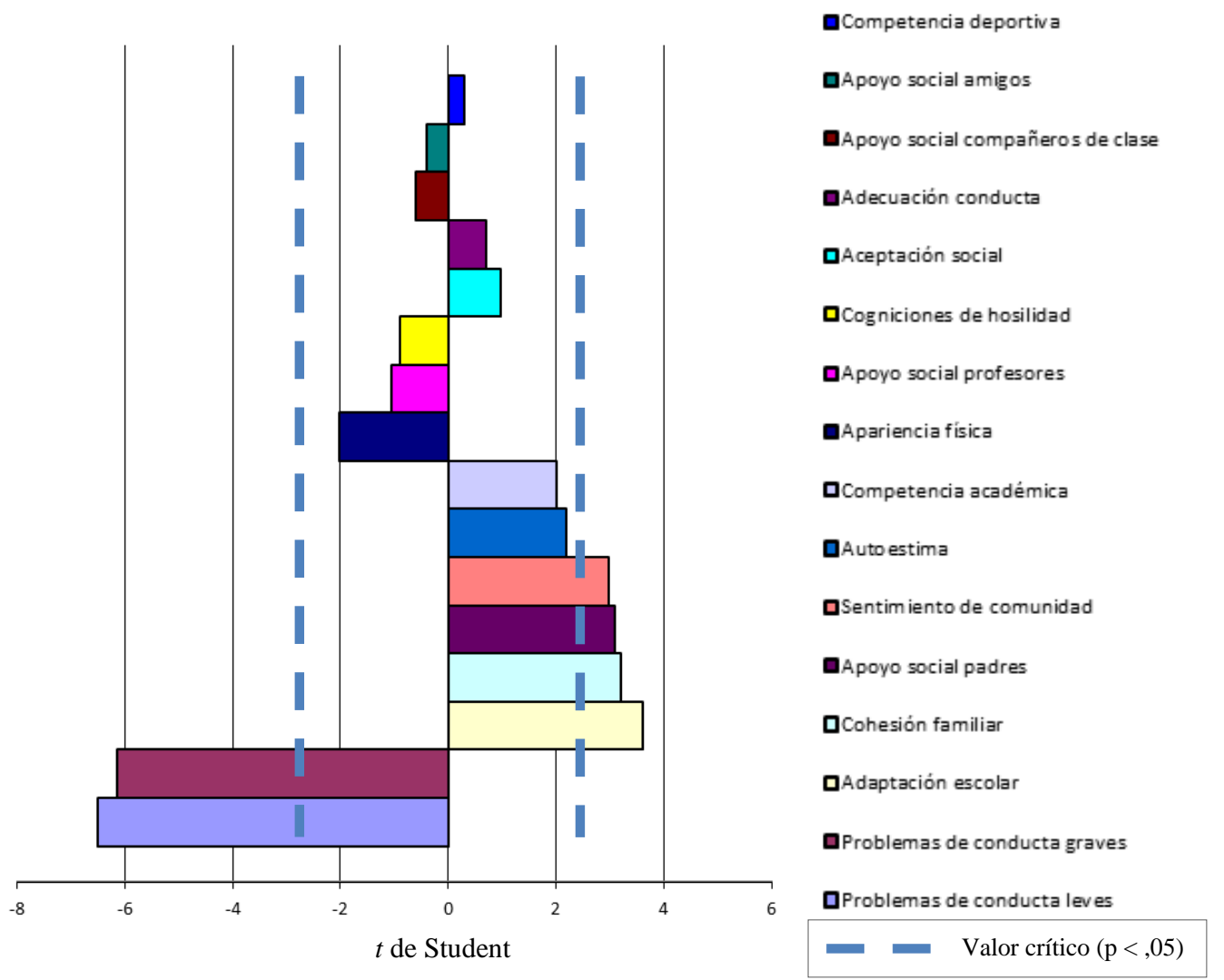

Figura 2. Varones adolescentes de educación secundaria adaptados (C2). 
En la figura 3, en tanto, se muestra que las adolescentes de $2^{\circ}$ y $4^{\circ}$ de secundaria (C3), de 13 y 15 años respectivamente, se caracterizan por estar significativamente por debajo de la media en la presencia de problemas de conducta graves, apariencia física percibida, competencia deportiva, competencia académica; mientras que se ubican por encima de la misma en apoyo social percibido de los/las amigos/as íntimos/as. También se situaron significativamente por debajo de la media en cogniciones de hostilidad y autoestima. Este grupo recibió el nombre de "mujeres de secundaria con baja autoestima".
Por su parte, el grupo mixto de varones y mujeres de todos los cursos (C4) de la figura 4 , se caracteriza por hallarse significativamente por encima de la media en problemas de conducta leves, cogniciones de hostilidad y por la presencia de problemas de conducta graves. Además, se encontraron por debajo de la media en la percepción de apoyo social de los padres, adecuación de la conducta, adaptación escolar, apoyo social de los profesores, apoyo social de los amigos, cohesión familiar, apoyo social de los compañeros, sentimiento de comunidad y autoestima. Este último grupo fue denominado "adolescentes en riesgo psicosocial".

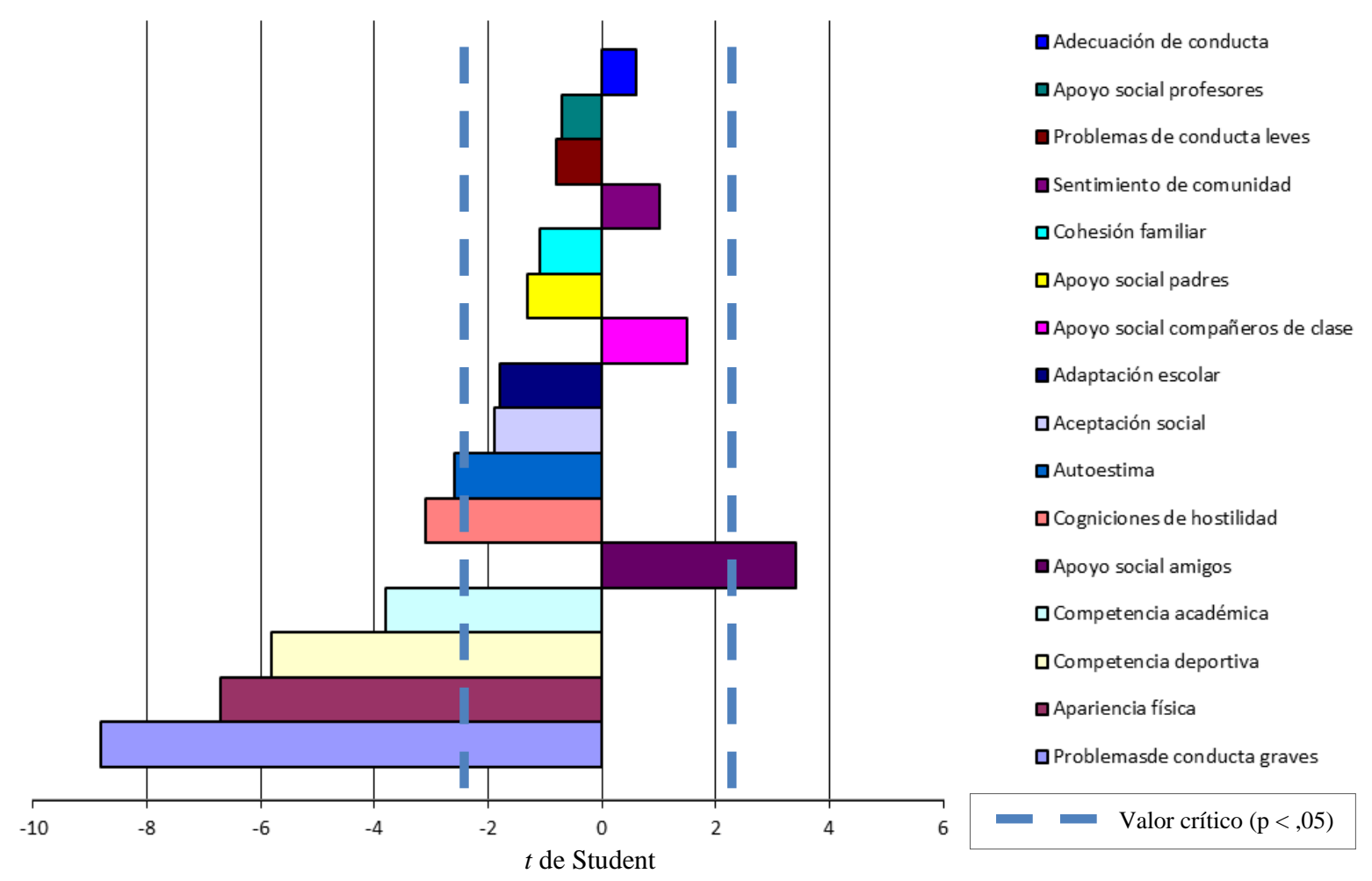

Figura 3. Mujeres adolescentes de educación secundaria con baja autoestima (C3). 


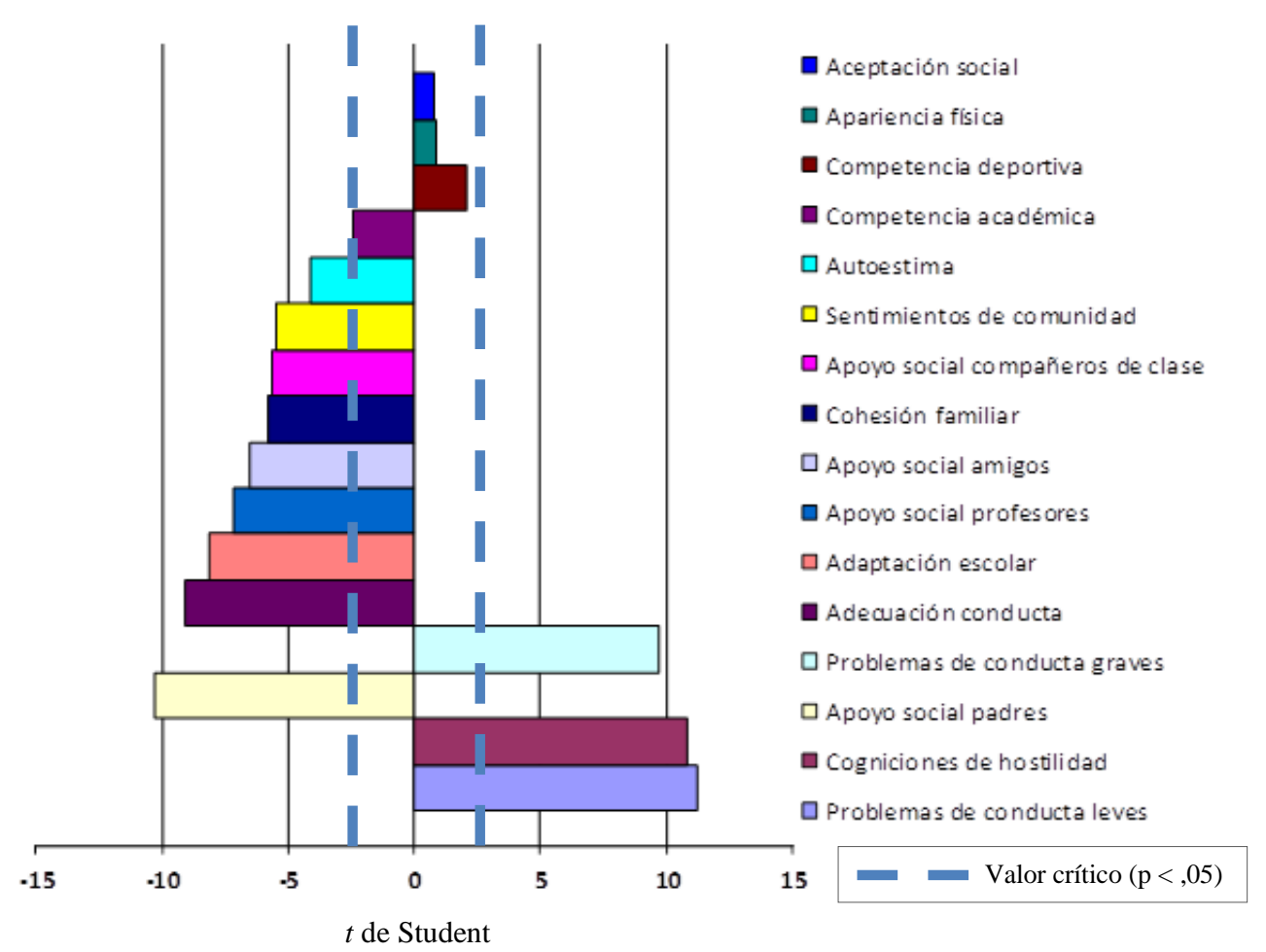

Figura 4. Adolescentes en riesgo psicosocial (C4).

La distribución de las características conductuales y psicosociales en los cuatro conglomerados aparece en la tabla 2; así como la contribución de la variable según el MANOVA $\left(\eta^{2}\right)$ y las diferencias significativas entre los grupos (Bonferroni). Los valores de la $\eta^{2}$ indicaron que las variables que explican en mayor medida las diferencias entre los grupos son: los problemas de conducta graves (,52) y leves $(, 47)$, el apoyo social de los padres $(, 19)$, la adaptación escolar $(, 19)$, las cogniciones de hostilidad (,18), la adecuación de la conducta $(, 13)$, la apariencia física
$(, 11)$, la cohesión familiar $(, 11)$, el sentimiento de comunidad (,09), el apoyo social de los amigos (,08), la autoestima $(, 08)$ y la competencia deportiva $(, 08)$.

Respecto de las diferencias en la puntuación total de ajuste psicosocial, el ANOVA realizado indicó diferencias significativas entre los grupos $(F(3,325)$ $\left.=104,32 ; p<, 001 ; \eta^{2}=, 34\right)$. La corrección de Bonferroni indicó diferencias significativas entre todos los grupos, quedando estos ordenados de $\mathrm{C} 1$ a $\mathrm{C} 4$, es decir, de mayor a menor ajuste. 
Tabla 2

Perfiles de los conglomerados

\begin{tabular}{|c|c|c|c|c|c|c|c|}
\hline \multirow{2}{*}{ Variables } & $\mathrm{C} 1$ & $\mathrm{C} 2$ & $\mathrm{C} 3$ & $\mathrm{C} 4$ & Global & \multirow{2}{*}{$\eta^{2}$} & \multirow{2}{*}{$\begin{array}{c}\text { Corrección de } \\
\text { Bonferroni }\end{array}$} \\
\hline & \multicolumn{5}{|c|}{$M(D T)$} & & \\
\hline $\begin{array}{l}\text { Problemas de } \\
\text { conducta } \\
\text { graves }\end{array}$ & $1,06(0,14)$ & $1,18(0,24)$ & $1,15(0,25)$ & $2,38(0,98)$ & $1,29(0,59)$ &, 52 & $\mathrm{C} 4>\mathrm{C} 1, \mathrm{C} 2, \mathrm{C} 3$ \\
\hline $\begin{array}{l}\text { Problemas de } \\
\text { conducta } \\
\text { leves }\end{array}$ & $1,17(0,24)$ & $1,37(0,43)$ & $1,57(0,63)$ & $2,89(1,03)$ & $1,58(0,79)$ & ,46 & $\begin{array}{c}\mathrm{C} 1<\mathrm{C} 2, \mathrm{C} 3, \mathrm{C} 4 \\
\mathrm{C} 2<\mathrm{C} 3, \mathrm{C} 4 \\
\mathrm{C} 3<\mathrm{C} 4\end{array}$ \\
\hline $\begin{array}{l}\text { Cogniciones } \\
\text { hostiles }\end{array}$ & $2,29(0,93)$ & $2,51(0,83)$ & $2,31(0,82)$ & $3,55(0,87)$ & $2,52(0,95)$ &, 18 & $\mathrm{C} 4>\mathrm{C} 1, \mathrm{C} 2, \mathrm{C} 3$ \\
\hline $\begin{array}{l}\text { Apoyo } \\
\text { social } \\
\text { amigos }\end{array}$ & $3,42(0,67)$ & $3,29(0,78)$ & $3,53(0,70)$ & $2,82(0,71)$ & $3,34(0,74)$ & ,08 & $\mathrm{C} 4<\mathrm{C} 1, \mathrm{C} 2, \mathrm{C} 3$ \\
\hline $\begin{array}{l}\text { Apoyo } \\
\text { social padres }\end{array}$ & $3,53(0,56)$ & $3,43(0,52)$ & $3,26(0,64)$ & $2,62(0,59)$ & $3,29(0,64)$ &, 19 & $\begin{array}{c}\mathrm{C} 4<\mathrm{C} 1, \mathrm{C} 2, \mathrm{C} 3 \\
\mathrm{C} 3<\mathrm{C} 1, \mathrm{C} 2\end{array}$ \\
\hline $\begin{array}{l}\text { Apoyo } \\
\text { social } \\
\text { compañeros }\end{array}$ & $3,27(0,57)$ & $3,19(, 55)$ & $3,23(0,54)$ & $2,84(0,59)$ & $3,18(0,59)$ &, 05 & $\mathrm{C} 4<\mathrm{C} 1, \mathrm{C} 2, \mathrm{C} 3$ \\
\hline $\begin{array}{l}\text { Apoyo } \\
\text { social } \\
\text { profesores }\end{array}$ & $3,02(0,72)$ & $2,85(0,73)$ & $2,81(0,72)$ & $2,38(0,56)$ & $2,82(0,73)$ & ,07 & $\begin{array}{c}\mathrm{C} 1>\mathrm{C} 3 \\
\mathrm{C} 4<\mathrm{C} 1, \mathrm{C} 2, \mathrm{C} 3\end{array}$ \\
\hline Autoestima & $3,27(0,56)$ & $3,16(0,57)$ & $2,93(0,59)$ & $2,76(0,70)$ & $3,06(0,62)$ & ,08 & $\begin{array}{l}\mathrm{C} 1>\mathrm{C} 3, \mathrm{C} 4 \\
\mathrm{C} 2>\mathrm{C} 3, \mathrm{C} 4\end{array}$ \\
\hline $\begin{array}{l}\text { Apariencia } \\
\text { física }\end{array}$ & $2,97(0,78)$ & $2,91(0,72)$ & $2,34(0,79)$ & $2,76(0,76)$ & $2,72(0,81)$ &, 11 & $\mathrm{C} 3<\mathrm{C} 1, \mathrm{C} 2, \mathrm{C} 4$ \\
\hline $\begin{array}{l}\text { Adecuación } \\
\text { de la } \\
\text { conducta }\end{array}$ & $3,01(0,63)$ & $3,06(0,50)$ & $2,90(0,55)$ & $2,35(0,57)$ & $2,90(0,60)$ &, 13 & $\mathrm{C} 4<\mathrm{C} 1, \mathrm{C} 2, \mathrm{C} 3$ \\
\hline $\begin{array}{l}\text { Competencia } \\
\text { académica }\end{array}$ & $2,97(0,64)$ & $2,86(0,59)$ & $2,58(0,68)$ & $2,59(0,58)$ & $2,76(0,65)$ & ,06 & $\begin{array}{l}\mathrm{C} 1>\mathrm{C} 3, \mathrm{C} 4 \\
\mathrm{C} 2>\mathrm{C} 3, \mathrm{C} 4\end{array}$ \\
\hline $\begin{array}{l}\text { Competencia } \\
\text { deportiva }\end{array}$ & $2,78(0,57)$ & $2,91(0,66)$ & $2,48(0,60)$ & $2,87(0,58)$ & $2,72(0,63)$ & ,08 & $\mathrm{C} 3<\mathrm{C} 1, \mathrm{C} 2, \mathrm{C} 4$ \\
\hline $\begin{array}{l}\text { Aceptación } \\
\text { social }\end{array}$ & $2,99(0,57)$ & $2,83(0,62)$ & $2,82(0,58)$ & $2,91(0,64)$ & $2,88(0,60)$ & ,01 & $\mathrm{C} 1>\mathrm{C} 3$ \\
\hline $\begin{array}{l}\text { Adaptación } \\
\text { escolar }\end{array}$ & $4,01(0,64)$ & $3,52(0,70)$ & $3,46(0,80)$ & $2,81(0,85)$ & $3,54(0,82)$ &, 19 & $\begin{array}{c}\mathrm{C} 1>\mathrm{C} 2, \mathrm{C} 3, \mathrm{C} 4 \\
\mathrm{C} 4<\mathrm{C} 2, \mathrm{C} 3\end{array}$ \\
\hline $\begin{array}{l}\text { Cohesión } \\
\text { familiar }\end{array}$ & $4,09(0,61)$ & $3,84(0,62)$ & $3,77(0,73)$ & $3,24(0,90)$ & $3,80(0,74)$ &, 11 & $\begin{array}{c}\mathrm{C} 1>\mathrm{C} 2, \mathrm{C} 3, \mathrm{C} 4 \\
\mathrm{C} 4<\mathrm{C} 2, \mathrm{C} 3\end{array}$ \\
\hline $\begin{array}{l}\text { Sentimiento } \\
\text { de comunidad }\end{array}$ & $3,88(, 65)$ & $3,52(, 64)$ & $3,64(, 68)$ & $3,21(, 73)$ & $3,62(, 70)$ & ,09 & $\begin{array}{c}\mathrm{C} 1>\mathrm{C} 2, \mathrm{C} 3, \mathrm{C} 4 \\
\mathrm{C} 4<\mathrm{C} 2, \mathrm{C} 3\end{array}$ \\
\hline
\end{tabular}

Nota: $M=$ Media; $D T=$ Desviación Típica; $\eta^{2}=$ Eta cuadrado.

Relación de los perfiles psicosociales con el malestar emocional

El MANOVA realizado con los indicadores de malestar emocional como variables dependientes mostró que estos reflejaban diferencias entre los cuatro grupos (Traza de Pillai $=, 23 ; F(12,891)=12,38 ; p<$ ,001; $\left.\eta^{2}=, 07\right)$. Los análisis univariados mostraron efectos significativos para las variables: ideación suicida, ánimo/afecto y energía/interés $(p<, 001)$. 
Los resultados del análisis discriminante realizado, con estas tres variables como variables canónicas, se presentan en la tabla 3. La primera función discriminante mostró la mejor combinación de los indicadores de malestar emocional que maximizaba la discriminación entre los grupos, explicando el $79 \%$ de la varianza. La segunda función explica un $20 \%$ de la varianza, mientras que la tercera no contribuye a discriminar entre los grupos. Asimismo, la primera función discriminante apareció positivamente relacionada con los tres indicadores considerados: ideación suicida $(r=, 88)$, energía/interés $(r=, 81)$ y ánimo/afecto $(r=, 79)$; mientras que la segunda mostró relaciones más moderadas con los tres indicadores: ideación suicida $(r=-, 47)$, energía/interés $(r=$ ,51) y ánimo/afecto $(r=, 22)$.

Los cuatro conglomerados encontrados difirieron en dos dimensiones (ver tabla 4). En la primera dimensión, que constituye una combinación de los tres indica- dores (alta ideación suicida, baja energía/interés y bajo ánimo/afecto), el grupo de mayor vulnerabilidad psicosocial (C4) obtuvo una puntuación más alta; mientras que disminuyó progresivamente la puntuación media obtenida en el resto de los grupos. En la segunda dimensión, que constituye una combinación fundamentalmente de baja ideación suicida y baja energía/interés, el grupo que obtuvo una mayor puntuación en contraste con los otros tres, es el de mujeres de secundaria con baja autoestima (C3), mientras que el que obtuvo una puntuación más baja es el grupo de mayor vulnerabilidad psicosocial (C4).

\section{Discusión}

El presente trabajo ha permitido identificar grupos naturales de adolescentes de una muestra representativa de Elche de entre 11 y 16 años, en función de algunas características sociodemográficas, conductuales y psicosociales.

Tabla 3

Resultados del análisis discriminante

\begin{tabular}{|c|c|c|c|c|c|c|c|c|c|}
\hline \multirow{2}{*}{$\begin{array}{c}\text { Función } \\
\text { discriminante }\end{array}$} & \multicolumn{3}{|c|}{$\begin{array}{l}\text { Coeficientes } \\
\text { estandarizados }\end{array}$} & \multirow[t]{2}{*}{ Autovalor } & \multirow{2}{*}{$\begin{array}{c}\% \\
\text { varianza }\end{array}$} & \multirow[t]{2}{*}{$p$} & \multicolumn{3}{|c|}{ Correlaciones } \\
\hline & IS & EI & AA & & & & IS & EI & $\mathrm{AA}$ \\
\hline 1 & 0,55 & 0,22 & 0,42 & , 18 & 79,74 & ,000 & ,88 & ,81 &, 79 \\
\hline 2 & $-1,07$ & 0,40 & 0,77 & ,04 & 20,32 & ,000 &,- 48 &, 51 & ,22 \\
\hline 3 & $-0,35$ & 1,29 & $-0,89$ &, 00 & 0,00 & 890 &,- 00 &,- 29 &, 56 \\
\hline
\end{tabular}

Nota IS = Ideación Suicida; EI = Energía/Interés; AA = Ánimo/Afecto.

Tabla 4

Medias de los conglomerados en las funciones discriminantes canónicas

\begin{tabular}{cccc} 
Conglomerados & F1 & F2 & F3 \\
\hline C1 & $-0,44$ & $-0,15$ & 0,00 \\
C2 & $-0,18$ & $-0,04$ & 0,00 \\
C3 & 0,16 & 0,28 & 0,00 \\
C4 & 0,93 & $-0,31$ & 0,00 \\
\hline
\end{tabular}

Nota: $\mathrm{F} 1$ = Función discriminante 1; F2 = Función discriminante 2; F3 = Función discriminante 3. 
Los resultados de este estudio han puesto de manifiesto que los distintos grupos encontrados difieren en una dimensión, de mayor a menor ajuste psicosocial.

En primer lugar, aparecen los adolescentes de $6^{\circ}$ de primaria (11-12 años) de ambos géneros $(\mathrm{C} 1)$, constituyendo el $29 \%$ de la muestra. Estos presentaron puntuaciones por encima de la media en la mayoría de los indicadores de ajuste psicosocial y por debajo en los comportamientos de riesgo.

En segundo lugar, se encuentra la mayoría de los varones de $2^{\circ}$ (13-14 años) y de $4^{\circ}$ de secundaria (15-16 años), que constituye el $27 \%$ de la muestra (C2). Estos también aparecen como un grupo con un buen ajuste psicosocial, aunque con puntuaciones más moderadas que el grupo anterior.

En tercer lugar, se encuentran las adolescentes de $2^{\circ}$ (13-14 años) y $4^{\circ}$ de secundaria (15-16 años), que fueron cerca del $35 \%$ de la muestra (C3). Al igual que sus coetáneos, estas tampoco manifestaron problemas conductuales importantes, aunque a diferencia de aquellos, presentaron un bajo autoconcepto físico, deportivo y escolar, una baja autoestima, combinado con una buena percepción del apoyo social de los/las amigos/as.

Por último, se identificó el grupo de mayor vulnerabilidad psicosocial (C4) integrado mayoritariamente por varones de los cursos superiores, aunque también algunas mujeres y algunos varones del resto de los cursos examinados; este constituyó el 14\% de la muestra. Los jóvenes de este grupo presentaron unas tasas más altas de problemas conductuales y hostilidad, así como puntuaciones por debajo de la media en la mayoría de las variables indicadoras de ajuste psicosocial.

Las características sociodemográficas y psicosociales de estos grupos encontrados coinciden, en gran medida, con los descritos por Carrasco (2004). Además, en consonancia con estudios previos y con la hipótesis 1 de este trabajo (ÁlvarezMonteserín et al., 1999; Bartlett et al., 2006; Bryant Ludden \& Eccles, 2007; Carrasco, 2004; Elzo Imaz, 2005; Weden \& Zabin, 2005; Zweig et al., 2001), la edad y el género desempeñaron un papel central a la hora de discriminar entre grupos de mayor a menor ajuste psicosocial en la adolescencia. De modo que, a grandes rasgos, los adolescentes más jóvenes presentaron mayor ajuste y con la edad aumentó la probabilidad de pertenecer a grupos de riesgo psicosocial, confirmándose, así, la hipótesis 2 de este trabajo. No obstante, cabe destacar que la mayoría de los adolescentes (tanto varones como mujeres) entre 11 y 16 años se mostró adaptados y apenas presentaron problemas de conducta.

Respecto del género, cabe resaltar las bajas puntuaciones en autoconcepto $\mathrm{y}$ autoestima en las adolescentes de mayor edad (13 y 15 años), no apareciendo asociadas a una mayor práctica de conductas antisociales o consumo de drogas. Resultados similares han sido hallados en otros trabajos con población española que emplearon el mismo instrumento (Pastor, Balaguer, \& García-Merita, 2003).

Una explicación plausible de este resultado pueden ser las prácticas de socialización familiar diferenciales por género. Así, por ejemplo, algunos estudios han informado de un mayor grado de monitorización parental en el caso de las adolescentes, 
comparativamente con sus compañeros (Raboteg-Scaric, Rijavec, \& BrajsaZcganec, 2001). Asimismo, en un estudio acerca de comunicación familiar encontraron que las familias con varones adolescentes empleaban en mayor medida intervenciones democráticas, mientras que aquellas con hijas adolescentes presentaban porcentajes más altos de expresiones autoritarias. Además, informaron que los varones adolescentes recibían más mensajes de apoyo de la familia, en comparación con las mujeres adolescentes, que recibían un mayor porcentaje de negativas que de mensajes de apoyo (Gimeno Collado, Anguera Argilada, Berzosa Sanz, \& Ramírez Ramírez, 2006). Por su parte, en comparación con las mujeres, un mayor porcentaje de varones se encuentra en el grupo de riesgo psicosocial, aunque la mayoría de ellos se halla en grupos con un buen ajuste psicosocial. Estos resultados permiten confirmar solo parcialmente la tercera hipótesis de este trabajo.

Considerando las variables psicosociales utilizadas como indicadores de ajuste, cabe señalar que han sido especialmente útiles para dibujar las fluctuaciones y características de esta población. Han permitido diferenciar subgrupos de forma significativa y describir sus características. Han sido especialmente ventajosas para discriminar y caracterizar a aquellos jóvenes que presentan una mayor vulnerabilidad y riesgo de presentar problemas de conducta importantes, indicando los puntos débiles y las líneas de intervención con estos.

Respecto del papel que juegan los indicadores de malestar emocional en la discriminación entre los cuatro grupos encontrados, los resultados de este estudio ofrecieron básicamente dos dimensiones. La primera constituyó una combinación de alta ideación suicida, baja ener- gía/interés y bajo ánimo/afecto, reflejando, de este modo, un alto malestar emocional. El grupo de mayor vulnerabilidad psicosocial obtiene una muy alta puntuación en esta dimensión, seguido a distancia del grupo de mujeres de secundaria con baja autoestima, mientras que los otros dos grupos presentan puntuaciones negativas.

La segunda dimensión comprendió una combinación fundamentalmente de baja ideación suicida y baja energía/interés. En esta, el grupo que obtiene una mayor puntuación, en contraste con los otros tres, es el de mujeres de secundaria con baja autoestima; y el que obtiene una puntuación más baja es el grupo de mayor vulnerabilidad psicosocial. Así, en consonancia con la hipótesis 4, los grupos de menor ajuste psicosocial manifiestan en mayor medida malestar emocional.

No obstante, las diferencias de género encontradas coinciden parcialmente con algunos resultados de la literatura que informan de la mayor presencia de problemas internalizantes en las mujeres $(\mathrm{Ca}-$ rrasco, 2004; Kramer et al., 2008) y externalizantes en los varones (Kramer et al., 2008). Según los resultados hallados en este estudio, las adolescentes manifestaron pocos problemas externalizantes y algunos internalizantes (baja autoestima y baja energía), mientras que solo aquellos adolescentes varones que mostraban importantes problemas externalizantes también presentaron mayores problemas internalizantes (alta ideación suicida, baja energía y bajo ánimo).

El presente estudio presenta algunas limitaciones que investigaciones futuras deberían remediar. Algunas de estas son: la utilización de medidas transversales, el estrecho rango de edad estudiado y la exclusión de otras muchas variables psi- 
cosociales que pueden aportar claridad para comprender mejor esta etapa, como por ejemplo, otras características familiares como el grado de comunicación, las creencias, los valores, la ilusión de invulnerabilidad o algunos rasgos de personalidad como la impulsividad. Asimismo, es importante tener en cuenta que el diseño correlacional de este trabajo no permite extraer conclusiones de causalidad.

A pesar de las limitaciones indicadas, se desprenden del presente trabajo dos importantes implicaciones prácticas. Por un lado, es posible detectar a aquellos jóvenes que se encuentran en una situación de riesgo y/o vulnerabilidad psicosocial, utilizando la batería de cuestionarios del presente estudio o similares. Por otro lado, cabe la posibilidad de diseñar programas específicos de intervención psicosocial y adoptar medidas preventivas ajustadas a las necesidades particulares de los distintos grupos de adolescentes.

Algunas recomendaciones podrían ser: intervenir en la mejora de las redes de apoyo social y en la percepción que los jóvenes tienen de estas; introducir programas de mejora de la autoestima y el autoconcepto en el contexto escolar, dirigidos a las adolescentes especialmente; concienciar de la necesidad de reducir la presión social hacia las adolescentes para ser atractivas y brillantes; fomentar la participación social de los/las adolescentes en su barrio para mejorar el sentimiento de comunidad; acercar el currículo académico a los intereses y necesidades de los/las jóvenes, para facilitar su adaptación escolar y dotarles de habilidades para el estudio; y por último, y no por ello menos importante, promover y mejorar la comunicación y la cohesión familiar.

\section{Referencias}

Álvarez-Monteserín, M. A., Martínez Arias, R., González, M., Chacón, F., Rojo, C., Rubio, A., ... Delgado, P. (1999). Risk factors in adolescence. Psychology in Spain, 3(1), 98103. Recuperado de http://www.psychologyinspain.com/content/reprints/1999/10.pdf

Atienza, F. L., Balaguer, I., \& Moreno, Y. (2002). El perfil de autopercepciones para niños: análisis de la validez factorial y la fiabilidad en la versión castellana. Psicothema, 14(3), 659-664. Recuperado de http://www.psicothema.com/pdf/779.pdf

Bartlett, R., Holditch-Davis, D., \& Belyea, M. (2005). Clusters of problem behaviors in adolescents. Research in Nursing \& Health, 28(3), 230-239.

http://dx.doi.org/10.1002/nur.20078

Bartlett, R., Holditch-Davis, D., Belyea, M., Halpern, C. T., \& Beeber, L. (2006). Risk and protection in the development of problem behaviors in adolescents. Research in Nursing \& Health, 29(6), 607-621.

http://dx.doi.org/10.1002/nur.20163

Benavides, G., Pastor, Y., Quiles, Y., Quiles, M. J., Terol, M. C., March, M. J., ... Pérez, V. (abril, 2004). Validación del Cuestionario de Dimensiones del Perfil de Depresión para Niños y Adolescentes. Trabajo presentado en VII Congreso Europeo de Evaluación Psicológica, Málaga, España. 
Bryant Ludden, A. \& Eccles, J. S. (2007). Psychosocial, motivational and contextual profiles of youth reporting different patterns of substance use during adolescence. Journal of Research on Adolescence, 17(1), 51-88.

http://dx.doi.org/10.1111/j.1532-7795.2007.00512.x

Buckner, J. C. (1988). The development of an instrument to measure neighborhood cohesion. American Journal of Community Psychology, 16(6), 771-791.

http://dx.doi.org/10.1007/BF00930892

Carrasco, A. -M. (2004). Consumo de alcohol y estilos de vida: una tipología de los adolescentes españoles. Revista de Psicología Social, 19(1), 51-79.

http://dx.doi.org/10.1174/021347404322726553

Crockett, L. J., Moilanen, K. L., Raffaelli, M., \& Randall, B. A. (2006). Psychological profiles and adolescent adjustment: A person-centered approach. Development and Psychopathology, 18(1), 195-214.

http://dx.doi.org/10.1017/S0954579406060111

Donnellan, M. B., Trzesniewski, K. H., Robins, R. W., Moffitt, T. E., \& Caspi, A. (2005). Low self-esteem is related to aggression, antisocial behavior and delinquency. Psychological Science, 16(4), 328-335.

http://dx.doi.org/10.1111/j.0956-7976.2005.01535.x

Elzo Imaz, J. (2005). Los adolescentes de hoy: consumo de alcohol y drogas y su relación con los comportamientos violentos. Revista de la Asociación Proyecto Hombre, 54, 814. Recuperado de

https://dialnet.unirioja.es/servlet/articulo? codigo=1446341

Estévez, C., Pastor, Y., Benavides, G., Quiles, Y., Quiles, M. J., Terol, M. C., ... Inglés, C. (abril, 2004). Validación del Cuestionario de Problemas de Conducta. Trabajo presentado en el IV Congreso internacional de psicología y educación: calidad educativa, Almería, España.

Feldstein, S. W. \& Miller, W. R. (2006). Substance use and risk-taking among adolescents. Journal of Mental Health, 15(6), 633-643.

http://dx.doi.org/10.1080/09638230600998896

Gavazzi, S. (2013). Theory and research pertaining to families with adolescents. En G. W. Peterson \& K. R. Bush (Eds.), Handbook of marriage and the family (pp. 303-327). New York, New York: Springer. http://dx.doi.org/10.1007/978-1-4614-3987-5_14

Gimeno Collado, A., Anguera Argilada, M. T., Berzosa Sanz, A., \& Ramírez Ramírez, L. (2006). Detección de patrones interactivos en la comunicación de familias con hijos adolescentes. Psicothema, 18(4), 785-790. Recuperado de http://www.psicothema.com/psicothema.asp?id=3309

Harter, S. (1985a). Manual for the self-perception profile for children. Denver, Colorado: University of Denver.

Harter, S. (1985b). Manual for the social support scale for children. Denver, Colorado: University of Denver. 
Harter, S. \& Nowakowski, M. (1987). Manual for the dimensions of depression profile for children and adolescents. Denver, Colorado: University of Denver.

Jackman, D. M. \& MacPhee, D. (2015). Self-esteem and future orientation predict adolescents' risk engagement. Journal of Early Adolescence, 1-28.

http://dx.doi.org/10.1177/0272431615602756

Jessor, R. (1993). Successful adolescent development among youth in high-risk settings. American Psychologist, 48(2), 117-126.

http://dx.doi.org/10.1037/0003-066X.48.2.117

Jiménez Gutiérrez, T. I., Musitu Ochoa, G., \& Murgui Pérez, S. (2005). Familia, apoyo social y conducta delictiva en la adolescencia: efectos directos y mediadores. Anuario de Psicología, 36(2), 181-195. Recuperado de

http://www.redalyc.org/articulo.oa?id=97017405002

Jiménez, T. -I., Musitu, G., \& Murgui, S. (2006). Funcionamiento y comunicación familiar y consumo de sustancias en la adolescencia: el rol mediador del apoyo social. Revista de Psicología Social, 21(1), 21-34.

http://dx.doi.org/10.1174/021347406775322214

Kelder, S. H., Hoelscher, D., \& Perry, C. L. (2015). How individuals, environments, and health behaviors interact: Social cognitive theory. En K. Glanz, B. K. Rimer, \& K. Viswanath (Eds.), Health behavior: Theory, research and practice (pp. 159-182). San Francisco, California: Jossey-Bass.

Kramer, M. D., Krueger, R. F., \& Hicks, B. M. (2008). The role of internalizing and externalizing liability factors in accounting for gender differences in the prevalence of common psychopathological syndromes. Psychological Medicine, 38(1), 51-61.

http://dx.doi.org/10.1017/S0033291707001572

Little, S. G., Akin-Little, A., \& Lloyd, K. (2013). School performance in adolescence. En W. T. O’Donohue, L. I. Benuto, \& L. W. Tolle (Eds.), Handbook of adolescent health psychology (pp. 223-234). New York, New York: Springer.

Mathieson, A. \& Koller, T. (Eds.). (2008). Social cohesion for mental well-being among adolescents. Copenhagen, Denmark: WHO Regional Office for Europe.

Mayberry, M. L., Espelage, D. L., \& Koenig, B. (2009). Multilevel modeling of direct effects and interactions of peers, parents, school, and community influences on adolescent substance use. Journal of youth and adolescence, 38(8), 1038-1049.

http://dx.doi.org/10.1007/s10964-009-9425-9

Musitu, G., Buelga, S., Lila, M. S., \& Cava, M. J. (2001). Familia y adolescencia: un modelo de análisis e intervención psicosocial. Madrid, España: Síntesis.

Olson, D. H., Portner, J., \& Lavee, Y. (1987). Family adaptability and cohesion evaluation scale [FACES III] (1985). En K. Corcoran \& J. Fischer (Eds.), Measures for clinical practice: A sourcebook (pp. 428-431). New York, New York: Free Press.

Pastor, Y., Balaguer, I., \& García-Merita, M. L. (2003). El autoconcepto y la autoestima en la adolescencia media: análisis diferencial por curso y género. Revista de Psicología Social, 18(2), 141-159.

http://dx.doi.org/10.1174/021347403321645258 
Pastor, Y., Balaguer, I., \& García-Merita, M. (2006). Relaciones entre el autoconcepto y el estilo de vida saludable en la adolescencia media: un modelo exploratorio por género. Psicothema, 18(1), 18-24. Recuperado de http://www.psicothema.com/psicothema.asp?id=3170

Pastor, Y., Balaguer, I., \& García-Merita, M. L. (2008). Testing a proposed model on the relationship between self-concept and a healthy lifestyle in adolescence. International Journal of Psychology Research, 3(1-2), 109-128. Recuperado de https://www.novapublishers.com/catalog/product_info.php?products_id=7150

Pastor, Y., Quiles, Y., \& Pamies, L. (2012). Apoyo social en la adolescencia: adaptación y propiedades psicométricas del "Social Support Scale for Children" de Harter (1985). Revista de Psicología Social, 27(1), 39-54.

http://dx.doi.org/10.1174/021347412798844060

Raboteg-Scaric, Z., Rijavec, M., \& Brajsa-Zcganec, A. (2001). The relation of parental practices and self-conceptions to young adolescent problem behaviors and substance use. Nordic Journal of Psychiatry, 55(3), 203-209.

http://dx.doi.org/10.1080/08039480152036092

Reeb, B. T., Chan, S. Y. S., Conger, K. J., Martin, M. J., Hollins, N. D., Serido, J., \& Russell, S. T. (2015). Prospective effects of family cohesion on alcohol-related problems in adolescence: Similarities and differences by race/ethnicity. Journal of Youth and Adolescence, 44(10), 1941-1953.

http://dx.doi.org/10.1007/s10964-014-0250-4

Reitz-Krueger, C. L., Nagel, A. G., Guarnera, L. A., \& Reppucci, N. D. (2015). Community influence on adolescent development. En T. P. Gullota, R. W. Plant, \& M. A. Evan (Eds.), Handbook of adolescent behavioral problems: Evidence-based approaches to prevention and treatment (pp. 71-84). New York, New York: Springer. http://dx.doi.org/10.1007/978-1-4899-7497-6_5

Sedikides, C. \& Gregg, A. P. (2003). Portraits of the self. En M. A. Hogg \& J. Cooper (Eds.), The SAGE handbook of social psychology (pp. 110-138). London, England: SAGE Publications.

Serrano, G., Godás, A., Rodríguez, D., \& Mirón, L. (1996). Perfil psicosocial de los adolescentes españoles. Psicothema, 8(1), 25-44. Recuperado de http://www.psicothema.com/pdf/3.pdf

Stattin, H., Romelsjö, A., \& Stenbacka, M. (1997). Personal resources as modifiers of the risk for future criminality: An analysis of protective factors in relation to 18-year-old boys. British Journal of Criminology, 37(2), 198-223.

http://dx.doi.org/10.1093/oxfordjournals.bjc.a014155

Trenz, R. C., Dunne, E. M., Zur, J., \& Latimer, W. W. (2015). An investigation of schoolrelated variables as risk and protective factors associated with problematic substance use among vulnerable urban adolescents. Vulnerable Children and Youth Studies, 10(2), 131-140.

http://dx.doi.org/10.1080/17450128.2015.1029034 
Vakalahi, H. F. (2001). Adolescence substance use and family-based risk and protective factors: A literature review. Journal of Drug Education, 31(1), 29-46.

http://dx.doi.org/10.2190/qp75-p9ar-nuvj-fjcb

Weden, M. M. \& Zabin, L. S. (2005). Gender and ethnic differences in the co-occurrence of adolescent risk behaviors. Ethnicity \& Health, 10(3), 213-234.

http://dx.doi.org/10.1080/13557850500115744

Welsh, W. N. \& Harding, C. (2015). School effects on delinquency and school based prevention. En M. D. Krohn \& J. Lane (Eds.), The handbook of juvenile delinquency and juvenile justice (pp. 181-198). London, England: Wiley-Blackwell.

http://dx.doi.org/10.1002/9781118513217.ch13

Zweig, J. M., Lindberg, L. D., \& McGinley, K. A. (2001). Adolescent health risk profiles: The co-occurrence of health risks among females and males. Journal of Youth and Adolescence, 30(6), 707-728.

http://dx.doi.org/10.1023/A:1012281628792

Zweig, J. M., Phillips, S. D., \& Lindberg, L. D. (2002). Predicting adolescent profiles of risk: Looking beyond demographics. Journal of Adolescent Health, 31(4), 343-353. http://dx.doi.org/10.1016/S1054-139X(02)00371-3 